\title{
INTERPRETATION OF RECENT HIGH ENERGY POLARIZATION DATA
}

\section{G. I. Kane}

Physics Department

University of Michigan

Ann Arbor, Mich. 48109

I am supposed to briefly discuss recent "high energy" measurements (say, above about two GeV/c) of spin observables. In the ayailable time I will comment on $C_{s s}, C_{n n}, P(n p \rightarrow n p)$, $\mathrm{P}\left(\pi_{\mathrm{p}} \rightarrow \pi \mathrm{p}\right), \mathrm{P}(\mathrm{pp} \rightarrow \mathrm{pp})$. The first three are ANI polarized beam measurements, the latter two $100 \mathrm{GeV} / \mathrm{c}$ polarized target Fermilab measurements. Finally I will comment on expectations at larger $t$ and very high energies.

It is necessary, of course, to describe the phase behavior of amplitudes if we want to understand polarization. I will discuss these in terms of $\mathrm{s}$-channel helicity amplitudes (SCHA) since they are presently the most well understood physically. I will speak in terms of scattering proceeding by exchanges with definite $t$-channel quantum numbers, modified by s-channel absorptive effects. Detailed treatment of these questions can be found in a recent review $\left.{ }^{1}\right)$. The relevent background on definitions of the observables, relations to different amplitudes, detailed references to experiments, etc, can be found in ref 2 or in various talks in the present proceedings.

\section{$\overline{\mathrm{C}_{\text {SS }}}$}

First consider the observale $\mathrm{C}_{\mathrm{SS}}$ or $\langle\mathrm{ss} 00\rangle$; see the talk of B. Sandler. This is one of the most interesting observables to measure. In terms of SCHA it is

$$
\mathrm{C}_{\text {SS }} \sim \operatorname{Re}\left(\varphi_{1}\left(\varphi_{2}+\varphi_{4}\right) *\right)
$$

where $\varphi_{1} \equiv\langle++|M|++\rangle, \varphi_{2} \equiv\langle++|M|--\rangle, \varphi_{4} \equiv\langle+-|M|-+\rangle$.

Observables fall into two categories. Some depend on combinations of amplitudes which behave in very characteristic ways, so reliable predictions should be possible. $\mathrm{C}_{\mathrm{SS}}$ is of this type. In addition, $\mathrm{C}_{\mathrm{SS}}$ is important because more than any other observable it distinguishes different models and approaches (see the discussions of Field and Stevens, and of Thomas, in ref 2). Qualitative success is required of any viable model. Other observibles such as $C_{n n}$ (below) depend on combinations of amplitudes such as differences of large 
numbers and are hard to predict.

To understand $C_{S S}$ we need $\varphi_{1}$ and $\varphi_{2}+\varphi_{4} \cdot \varphi_{1}$ is the dominant elastic applitude, mainty diffractive and imaginary at small t. Then it rotates clockwise until Imp has a zero for $-t \approx 1 \mathrm{GeV}^{2}$ at ANL energies.

Two points are important about $C_{2}+\varphi_{4}$, which is dominated by $\pi+B$ exchange quantum numbers. FIrst, it has an imaginary part of the same sign as $\varphi$; this follows from the Regge behavior of $\pi$ and $B$ plus approximate exchange degeneracy. The relative size of $B$ to $\pi$ is well determined (1) by the measurement of $\sigma_{T}(\uparrow \downarrow)-\sigma_{T}(\uparrow \uparrow)$. Second, $\operatorname{Re}\left(\varphi_{2}+\varphi_{4}\right)$, which is clominated by the $\pi$ exchange, is given mainly by ( $2 \mathrm{x}$ pion pole - absorption) and has two zeross as shown in fig 1.

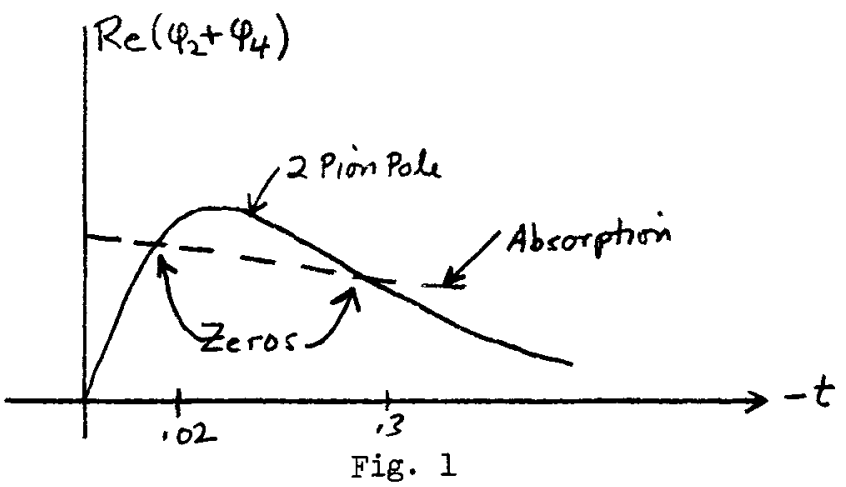

The first of these is the well-known zero causing the sharp $\pi$-exchange peak in $n p+p n, y p \rightarrow \pi^{+} n$, etc; the second is harder to isolate, and may be best tested here. It is a crucial prediction of the absorption approach $(\mathbf{1})$.

These arguments give $\varphi_{1}$ rotating through the 3 ra quadrant as - $t$ increases, largely parallel to $\varphi_{2}+\varphi_{1}$ over most of the range, with a significant real part for the product, and allow one to understand the prediction. It is shown, along with the new measurements that are largely consistent, in Sandler's talk.

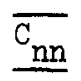

Next briefly consider $\mathrm{C}_{\mathrm{nn}}$. It is

$$
C_{n n} \sim 2\left|\varphi_{5}\right|+\left(\operatorname{Re} \varphi_{1}\right) \operatorname{Re}\left(\varphi_{2}-\varphi_{4}\right)-\operatorname{Im} \varphi_{1} \operatorname{Im}\left(\varphi_{3}-\varphi_{4}\right)
$$


All the separate pieces are large and lots of cancellations occur. Consequently the $s$ and $t$ dependence will not be those of any simple contribution; typical Regge s-dependence will not appear even though the individual terms have it. The zero structure will. not come directly from absorption but from cancellations. The model predictions are not in very good agreement with the data (and it would have been fortuitous if they were), but the qualitative structure is there, as shown in fig 2. The second zero, which is a difference of two large numbers, needs to move in about $1 / 2 \mathrm{GeV}^{2}$; then the curve will move up for $-t>1 \mathrm{GeV}^{2}$ and look very much like the data. At smaller $t$ the height is again a difference of large numbers and could be adjusted to agree.

Thus observables like $C_{S S}$ test the theory well, while those like $C_{n n}$ are only somewhat restrictive, but, conversely, provide good measurements of coupling constants to get the cancellations right. The former are useful to gain confidence in the model and the latter to measure new quantities.

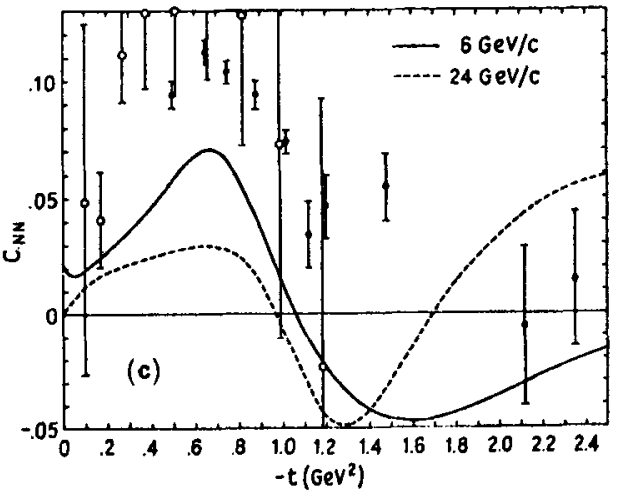

Fig. 2

$$
\underline{n p \rightarrow n p}
$$

Now turn to $n p$ elastic scattering. There are mainly two considerations of interest. First the sign, relative to $\mathrm{pp}$. If only $I=1$ exchanges are important in the flip amplitude, then the polarization for $n p$ would be opposite in sign to $p p$. optical models would have it the same. When the original experiment was done the size of the isoscalar flip couplings ( $w, f$ exchange) were not known. There were indications $(3)$ which allowed people who believed them to guess the qualitative behavior of the polarization. The observed sign at small $t$ and detailed shape in fact give the best way to determine these couplings. (1). 
The energy dependence is a different problem, with $P$ varying rapidly from 3 to $6 \mathrm{GeV} / \mathrm{c}$ experimentally but not theoretically. If the data is approximately described by the theory at $12 \mathrm{GeV} / \mathrm{c}$ it will be clear that the discrepancy at $3 \mathrm{GeV} / \mathrm{c}$ is due to a low lying contribution which is of interest in its own right but not directly relevant to our understanding of the high energy amplitudes.

\section{$100 \mathrm{GeV} / \mathrm{c}$ and High Energies}

Now there is preliminary data for a FNAL polarized target experiment on $\pi^{ \pm p}$ and $\mathrm{pp}$ at $100 \mathrm{GeV} / \mathrm{c}$; see the talk of R.KIine.

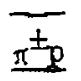

For $\pi^{ \pm}$first note that the mirror symmetry is approximately maintained, which puts a limit on the amount of $f$ helicityflip coupling since the isovector $\rho$ must dominate. It is not a strong limit, however; a ratio for the $f G_{+-} / G_{++} \simeq 1 / 4$ still leaves $(1)$ the mirror symmetry largely intact.

Second, notice that the zero may be moving closer to $t=0$ as $\mathrm{P}_{\mathrm{I}}$ increases but it is not moving very rapidly if it is. It remains near $-t \approx 0,5 \mathrm{GeV}^{\mathrm{a}}$.

\section{$\overline{p p+p p}$}

Since most high energy data will involve polarization for $p p \rightarrow p p$, I will discuss 1ts behavior in some detail. Even more detail is available in ref 1 and 4 .

At high energies it is a satisfactory approximation to put

$$
P \propto \operatorname{Im}\left(\varphi_{1} \varphi_{5}^{*}\right)
$$

and to see qualitative behavior,

$$
P \propto\left(\operatorname{Im} \varphi_{1}\right)\left(\operatorname{Re} \varphi_{5}\right)
$$

so one can think in terms of a dominantly imaginary non-flip amplitude $\varphi_{1}$ and a dominantly real flip amplitude $\varphi_{E^{\circ}}$.

First consider $\operatorname{Im} \varphi_{1}$. It has a pomeron contribution essentially constant in $s$, plus Reggeons that fall about as $1 / \sqrt{s}$. They are sketched in $t$ at two different energies in fig 3 . 


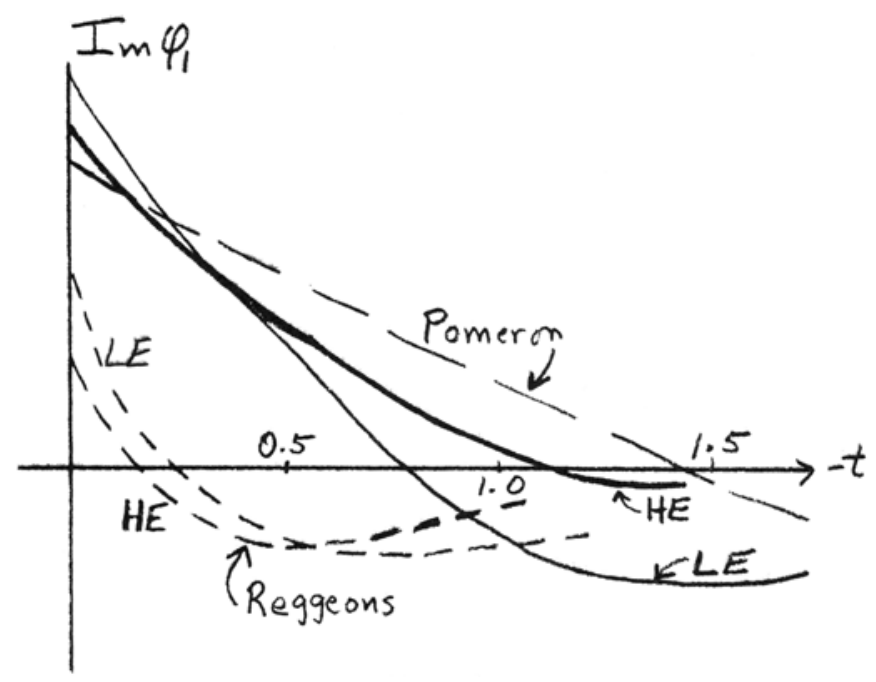

Fig. 3

The dashed lines are Reggeon contributions, with a zero near $-t \simeq 0.25 \mathrm{GeV}^{2}$. As $s$ increases the size of the Reggeon contribution decreases and shrinkage moves the zero a little toward $t=0$. The Pomeron contribution has the zero near $-t=1.4 \mathrm{GeV}^{2}$ which gives the pronounced dip at ISR energies. Beyond its zero the Reggeon interferes destructively with the Pomeron and at lower energies (LE) gives a zero in the full amplitude near $-t=0.8 \mathrm{GeV}^{2}$. At higher energies (HE) the Reggeon is smaller in size so it does not cancel the pomeron until further out in $-t$, and the zero in $\operatorname{Im} \varphi_{1}$ moves out as s increases.

Next gonsider $\operatorname{Re}_{.5}$. This behaves as a standard flip amplitude (1) and looks as in fig 4 , with a zero near. $-t=0.7 \mathrm{Gev}^{2}$ at $L E$ which moves toward $t=0$ as $s$ increases.

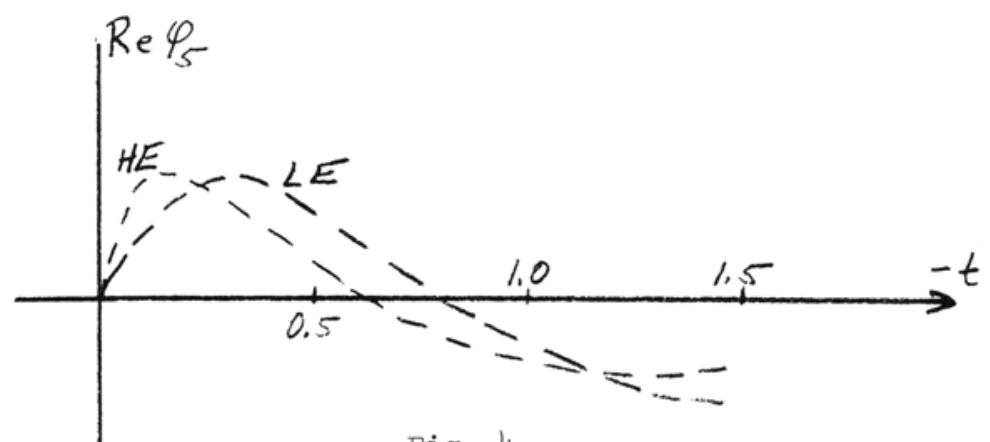

Fig. 4 
Thus at low energies ( $10 \mathrm{GeV} / \mathrm{c})$ the product $\operatorname{Im} \varphi_{1} \operatorname{Re} \varphi_{5}$ has two nearby zeros and $\mathrm{P}$ has a "double zero" structure. As $s$ increases, the zero in Imc, moves out, the zero in Re $\varphi_{5}$ moves slowly in, and $P$ goes negative between the two zeros, so one expects polarization as in fig 5 .

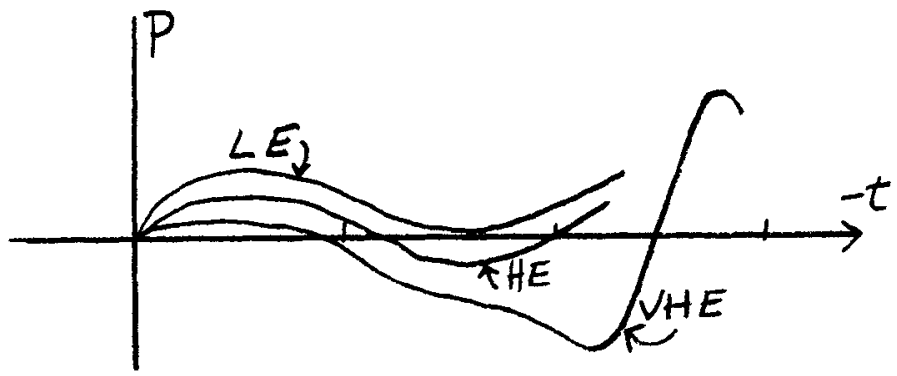

Fig. 5

In the dip region at very high energies (VHE) one has $\varphi_{5}$ mainly negative and real, and $\varphi_{1}$ rotating from large nègative imaginary to large positive imaginary, so $P$ will be large near the dip.

There is one more effect that must be included at higher energies (say above about $40 \mathrm{GeV} / \mathrm{c}$ ) to get a quantitative description $(4)$. Just as unitarity builds up diffraction dissociation, where the mass of an external particle changes, it may build up a Pomeron helicity flip amplitude. In particular, we expect this (4) from the long range contribution associated with two pions in the imaginary part of the amplitude,

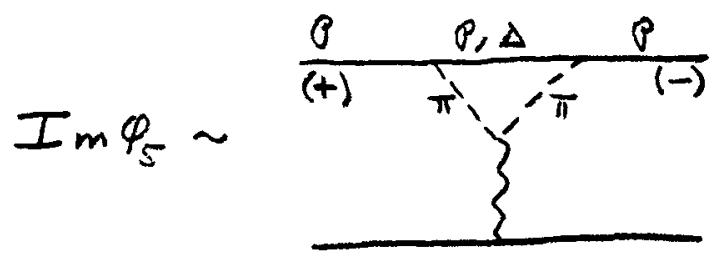

Fig. 6

At large impact parameter this will not be significantly suppressed or modified by other more central contributions or absorption. To make an explicit model we assume (see ref 4 for details) 


$$
\operatorname{Im} \varphi_{5}(t) \sim J_{1}(R /-t)
$$

and fix the size by requiring agreement at large impact parameter with the contribution of fig 6 . This will persist to high energies, only differing by powers of $\mathrm{ln} s$ from the Pomeron.

Since this will generally have different energy dependence from the non-flip Pomeron and the same signature, it will generally have a different phase $\left({ }^{4}\right)$ and polarization is generated for $p p \rightarrow p p, \Lambda p \rightarrow \Lambda p$, etc. We expect it will look as in fig 7 .

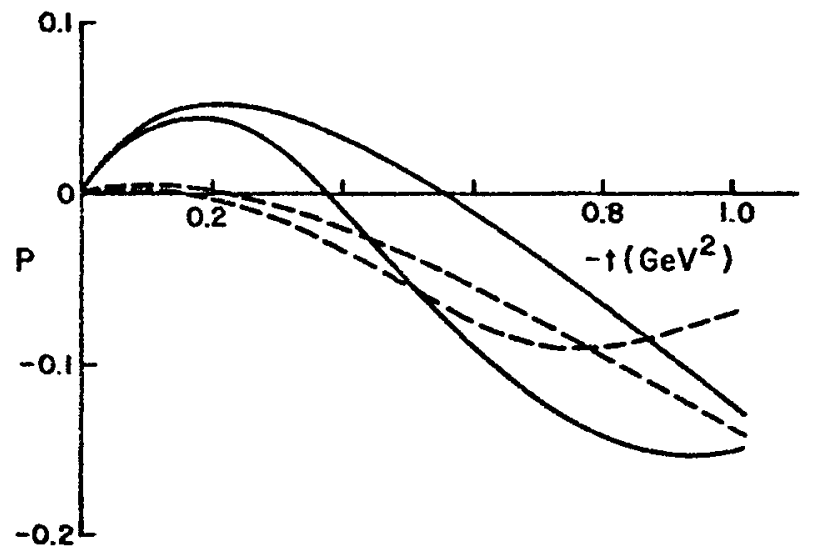

Fig. 7

Unfortunately, the sign of this contribution is not trivial to determine. In ref 4 we assume it would be the observed sign at small t. H. Navelet (private communication) has pointed out that the negative sign of $P\left(\pi^{+} p\right)+P\left(\pi^{-} p\right)$ at $100 \mathrm{GeV} / \mathrm{c}$ suggests a negative sign for our diffractive contribution. A definitive calculation should be possible but has not been done yet.

Now turn to the high energy pp data. The data at 45 $\mathrm{GeV} / \mathrm{c}$ from Serpukhov (5) indeed behaves as expected, with a zero moved in to $-t=0.5 \mathrm{GeV}^{2}$. However, it is not clear whether the $100 \mathrm{GeV} / \mathrm{c}$ data is correctly described (see the talk of R. Kline for the data).

Basically, $P$ is smaller at small t than expected. [B. Wicklund has emphasized, however, that a calculation putting in the correct low-lying isoscalar exchange at low energies requires less of the standard Reggeon contribution, so the descrepancy may be smaller in a more comprehensive calculation.] Also, the zero may be moved into smaller $t$ than expected. Bot,h of 
those would indeed occur if our diffractive polarization was opposite in sign to the Reggeon contribution (so it would have the sign for which Navelet argues). It is not likely that the Reggeon zero is moved in to $0.3 \mathrm{GeV}^{2}$; recall that it did not shift much in $\pi \pm \mathrm{p}$. When the data is firm and there has been time to do the theory carefully the situation should be clear. In any case, the polarization will still be large at FNAL energies in the dip region.

\section{CONCLUDING REMARKS}

For the future at high energies a new possibility seems to be opening up. Recent measurements at the ISR have suggested that cross sections are much larger at $3 \leqslant-t<8 \mathrm{GeV}^{2}$ than expected, perhaps two or more orders of magnitude larger (U. Sukhatme, private communication). Polarization measurements are being attempted in the dip region now, and the cross section decrease out to $-t \sim 4-5 \mathrm{GeV}^{8}$ is little enough that apparently $P$ should be measureable there as well. Why is $\mathrm{d \sigma} / \mathrm{dt}$ so large there? It is hard to see how conventional conceptions of geometrical hadron behavior could give such a large cross section. Possibly the constituent nature of hadrons is showing up at surprisingly small $t$ and can be probed in a new way by polarization measurements there.

\section{$\overline{\text { REFERENCES }}$}

1. G.L. Kane and A. Seidl, Rev. Mod. Phys. 48309 (1976)

2. ANL-HEP-CP-75-73 "Physics with Polarized Beams", Report of the Technical Advisory Panel.

3. F. Gault, A.D. Martin, and G.L. Kane, Nuc. Phys. B 32419 (1971).

4. J. Pumplin and G.L. Kane, Phys. Rev. D1l 1183 (1975)

5. A. Gaidot et. al., Phys. Lett. 613 103 (1976) 